\title{
Quality improvement, research, and evidence-based practice: 5 years experience with pressure ulcers
}

"The first requirement of a hospital is that it should do the sick no harm." Florence Nightingale (1863)

Skincare, a fundamental component of basic patient care, reflects on the overall quality of care that a patient receives in hospital. Quality care aimed at preventing and minimising skin breakdown and pressure ulcers has been identified as a nursing research priority. ${ }^{1}$ During the past 5 years, we have undertaken a large research utilisation effort focused on skincare and pressure ulcers involving more than 20 nursing units at a Canadian university affiliated teaching institution. Diverse activities were undertaken to address the barriers to evidence-based practice with efforts occurring across the setting and at many levels of the organisation.

\section{Starting with a clinical problem}

The initiative began in 1993 when an increase in the incidence of pressure ulcers was perceived in our setting. There had been more referrals to the enterostomal therapist and to plastic surgery, and sporadic reporting by clinical staff of their suspicions that skin breakdown was increasing in their areas. At that time no unit based mechanism for capturing reliable data on pressure ulcers was in place nor was it possible to retrieve data from the hospital information system.

Even though it is a quality issue, the pressure ulcer initiative was set in a larger context of professional practice by linking it to the existing nursing quality improvement, research, and education infrastructures. The nurse specialists for quality improvement and research and evaluation took the lead on the project with support from several clinical nurse educators and managers. Critical to the success of the project was the support of the chief nursing officer who was vice president of patient services.

A multidisciplinary task force developed and implemented a skincare programme. Two key initiatives ran concurrently: design and implementation of skincare programmes on the clinical units and systematic assessment of pressure ulcer prevalence. The skincare task force, led by the director of nursing research and quality improvement, reviewed the research and existing evidence-based clinical guidelines such as the 1992 US Agency for Health Care Policy and Research (AHCPR) Clinical Practice Guideline on pressure ulcers in adults. $^{2}$

The task force considered the AHCPR practice guideline to be a credible source. Convened by a prominent health association, the guideline was developed by a multidisciplinary panel which reviewed the research and graded the evidence. It offered a standard for evidence-based practice. Although fortunate to have a current clinical practice guideline available, most of the guideline recommendations were supported by level $\mathrm{C}$ evidence indicating that they were drawn from expert opinion and panel consensus in the absence of research.
It was essential to evaluate the guideline for use in our setting because it would be the foundation of our approach to improve quality of care. ${ }^{3}$ An important component of care was the assessment of risk, followed up by the selection of evidence-based prevention strategies. The Braden Scale, 1 of 2 tools recommended in the AHCPR guideline, was selected to assess risk of pressure ulcers in a standardised manner. ${ }^{4}$ The scale has an overall risk score derived from 6 subscales: sensory perception, moisture, activity, mobility, nutritional status, and friction/shear which are scored from 1 (most at risk) to 3 or 4 (least at risk) for a maximum total score of 23 points. A risk cutoff score of $\leqslant 16$ indicates risk for pressure ulcer development in acute care populations. ${ }^{4}$ However, the guideline stressed that the specific cutoff scores should be evaluated and assigned within the context of individual settings.

The task force recommended that the following issues be addressed in planning for effective care and prudent use of resources:

- Baseline assessment of the extent of the problem

- Evaluation of the accuracy of a risk assessment method

- Practice changes including development of an educational strategy to upgrade nursing skills in pressure area assessment using grading or staging of sores

- A method for ongoing monitoring.

Understanding the extent of the local problem

A 12 hour prevalence survey was done on all inpatient units to establish risk of pressure sore development and the proportion of patients with skin breakdown. Volunteer clinical nurses collected the data after instruction about evidence-based practice, clinical practice guidelines, and how to assess prevalence. After a 2 week follow up period, all new occurrences of pressure ulcers were documented and the accuracy of a risk assessment scale was evaluated.

\section{Evaluation of risk method}

Our evaluation of the sensitivity and specificity of the Braden Scale was less favourable than previously reported. ${ }^{56}$ Others have also assessed the reliability and validity of the Braden total score, as well as the importance of specific risk factors. ${ }^{7}$

\section{Changes in practice}

The AHCPR guideline provided standards of care for documenting risk, assessing skin, and staging ulcers. The prevalence and incidence studies provided evidence for planning the clinical programme.

Given our results using the Braden Scale total score, the task force did not feel that its accuracy in our setting was acceptable as a basis for initiating prevention strategies and assigning resources. ${ }^{9}$ Instead, we proceeded with caution and 
used the Braden risk subscales. A multidisciplinary group (which included nurses, physiotherapists, occupational therapists, and a nutritionist) developed the interventions. Each evaluated their discipline specific literature and agreed upon a set of actions to respond to patient deficits in moisture, sensory perception, activity, mobility, nutritional status, and friction/shear. For example, an algorithm using the mobility and activity subscales directed the use of pressure relieving products and devices. Clinical managers and directors negotiated allocation of special beds and surfaces. Consulting practices were modified to allow direct referral by nurses to physiotherapy or occupational therapy for skin issues. This previously had required a physician's order. Currently, work is underway to incorporate skin assessment into the hospital care mapping process.

Although skincare and pressure ulcer management is considered to be a nursing care issue, many hospital systems contribute to problem solution (eg, accessibility of linen and supplies, quality nutritional services, and availability of porters and orderlies). Thus, it was important to communicate beyond the nursing staff. Through this process, we compensated for shortcomings in the clinical practice guideline and started our skincare initiative with wide participation in the practice guideline evaluation and the pressure ulcer prevalence (PUP) study.

\section{Monitoring the problem}

The annual PUP study continued for several years. The task force measured annual progress and compared our rates with other published rates. Pressure ulcer prevalence has declined for all stages of sores from $32.3 \%$ in 1993 to $19.6 \%$ in 1997. Rates of ulcers at more serious stages ( $\geqslant$ stage 2 ) have remained fairly constant. Problem prediction and prevention has improved-the proportion of stage 1 ulcers (persistent redness) was decreased from 18\% to $9 \%$ over 4 years. This decrease occurred despite an increase in the proportion of patients $>80$ years of age, a population at high risk of skin breakdown.

In addition to annual monitoring, an indepth trend analysis was done after 4 years. The nurse specialist for research convened a panel of clinical and managerial staff to analyse and interpret the findings. Their report recommended a focus on institution-wide risk management for identified populations; unit based incidence data collection; and intervention studies.

To increase the potential use of our study results, our reports were reader friendly, user specific, and tailored to programme, nurse director, and manager needs. Classes and individual consultations were provided to assist staff with interpretation of their unit data. The evidence was used for policy and clinical decision making.

The PUP study results are used as the basis of a hospital outcome indicator. By linking the research utilisation initiative to the quality improvement mandate, resources have been available to continue the pressure ulcer work. In addition, other studies have evolved from the comprehensive nursing data set.

\section{Linking quality improvement and research to promote evidence-based practice}

Over 5 years, we have addressed the central barriers to research utilisation: quality of research findings and organisational and individual factors. ${ }^{10}$ The task force activities and the prevalence studies were promoted at all levels of the organisation using written and verbal presentations. The vice president of patient services worked at the board of trustee and executive level, whereas others advanced the activity with their peers (eg, educators and clinical managers). The PUP surveyors and the newly installed skincare monitors, who were clinical nurses on each unit, acted as "idea champions" to reinforce awareness of pressure ulcer activity. Newsletters provided details of the studies, and papers were presented at annual nursing research and quality improvement conferences and external scientific meetings. Manuscripts were published and circulated throughout the clinical areas. ${ }^{911}$ Funds were sought and obtained annually through corporations, hospital foundations, and hospital volunteer programmes. The skincare programme and the supporting research have become more credible.

In year 2, we linked our work with a sister teaching hospital to expand our knowledge of the prevalence of pressure ulcers in tertiary settings. ${ }^{11}$ Currently, we are participating in a 3 year Province-Wide Nursing Project (PWNP) funded by the Ontario Ministry of Health aimed at best practice in nursing. In this project we continued the focus on evidencebased skincare, particularly prevention of pressure ulcers with continuity across a tertiary care hospital, a long term care setting, and the community. These collaborative linkages allow us to continue to promote pressure ulcer initiatives and evidence-based practice. In the PWNP, we have used the Ottawa model of research use. ${ }^{10}$

\section{Lessons learnt}

The nature of the work is long term and iterative rather than linear. It requires diverse approaches to overcome organisational and individual level barriers to using research evidence. Linking research use to quality improvement work was important. The comprehensive data set from the PUP study has provided a benchmark against which to evaluate changes in care as well as an ongoing source of evidence for planning care activities. The nursing data set has also generated scholarly interest in further research in areas such as the profiling of particular populations (eg, neurological patients). Based on our experience, we offer the following observations:

- Responsibility and accountability should be assigned for all aspects of the process

- National and international clinical practice guidelines should be evaluated and adapted to the local setting. This increases knowledge about the appropriateness of guideline recommendations, and the process itself assists local "buy-in"

- Identification of barriers to evidence-based practice is essential

- The process to overcome these identified barriers should be strategic and involve acquiring support and resources and involving people at relevant organisational levels. Application of a research use model may be helpful

- An evidence gathering and monitoring process can show the importance of the issue and provide leverage for accessing resources

- Reporting and communicating in user friendly, audience specific formats to the different stakeholders and decision makers from clinical to corporate levels facilitate use of the evidence

- Organisational and practice change is incremental. It is useful to set realistic short, mid range, and long term goals and time lines.

\section{Conclusion}

The path to evidence-based practice is complex, fraught with pitfalls, and requires extraordinary effort and dedication to complex processes for success. It requires systematic planning that is flexible enough to meet the challenges involved in changing the practice environment. For us, this included massive organisational redesign and healthcare 
restructuring. Our process of implementing a practice guideline as a standard of care presented numerous challenges and necessitated the engagement of clinical, quality improvement, and research expertise. Our institution has recently been amalgamated with 3 other institutions and, with this initiative, we continue our efforts to provide evidence-based nursing care from a sound foundation.

Nurse Specialist, Research and Evaluation

MARGARET B HARRISON, RN, PhD Ottawa Hospital Civic Campus,

Principal Investigator, Loeb Research Institute,

Clinical Associate, University of Ottawa,

Ottawa, Ontario, Canada

Assistant Professor, University of Ottawa, Ottawa, Ontario, Canada

JO LOGAN, RN, PhD

Clinical Nurse Specialist, Neuroscience,

LYNN JOSEPH, RN, BScN

Ottawa Hospital Civic Campus,

Ottawa, Ontario, Canada

Principal Investigator, Loeb Research Institute,

IAN D GRAHAM, $\mathrm{PhD}$

Assistant Professor, Faculty of Medicine,

University of Ottawa,

Ottawa, Ontario, Canada
The authors wish to acknowledge the hard work and dedication of the nurse surveyors, enterostomal therapists, and team leaders during this project. The enormous commitment from the vice president of patient services, the nursing directors, nurse educators, and the quality improvement nurse specialists has ensured that this nursing research has become integral to professional practice in our setting.

1 Dennis KE, Howes DG, Zelauskas B. Identifying nursing research priorities: a first step in program development. Appl Nurs Res 1989;2:10813 .

2 Agency for Health Care Policy and Research. Pressure ulcers in adults: prediction and prevention. Rockville, MD: US Department of Health and Human Services, 1992.

3 Basinski AS. Evaluation of clinical practice guidelines. CMAJ 1995;153: $1575-81$.

4 Bergstrom N, Demuth PJ, Braden BJ. A clinical trial of the Braden Scale for predicting pressure sore risk. Nurs Clin North Am 1987;22:417-28.

5 Bergstrom N, Braden BJ, Laguzza A, et al. The Braden Scale for predicting pressure sore risk. Nurs Res 1987;36:205-10.

6 Braden BJ, Bergstrom N. Predictive validity of the Braden Scale for pressure sore risk in a nursing home population. Res Nurs Health 1994; 17:459-70.

7 Maklebust J, Magnan MA. Risk factors associated with having a pressure ulcer: a secondary data analysis. Adv Wound Care 1994;7:25,27-8,31-4.

8 Salvadalena GD, Snyder ML, Brogdon KE. Clinical trial of the Braden Scale on an acute care medical unit. J ET Nurs 1992;19:160-5.

9 Harrison MB, Wells G, Fisher A, et al. Practice guidelines for the prediction and prevention of pressure ulcers: evaluating the evidence.J App Nurs Res 1996;9:9-17.

10 Logan J, Graham I. Toward a comprehensive interdisciplinary model of health care research use. Science Communication, in press.

11 Fisher A, Denis N, Harrison MB, et al. Quality management in skin care: understanding the problem of pressure ulcers. Canadian Journal of Quality in Health Care $1996 ; 13: 4-11$.

\section{Invitation to submit letters and papers on implementation}

4 vidence-Based Nursing provides practising nurses with the best research evidence along with advice from clinical Cexperts regarding how this evidence, combined with patient preferences, can be applied to practice. We hope that Evidence-Based Nursing will make an important contribution to nursing, and ultimately to patient care, by bringing the findings of rigorous research to the attention of nurses, by promoting the critical appraisal of research, and by fostering implementation.

An important step in the practice of evidence-based nursing is the sharing of successful implementation strategies. We therefore welcome submission of manuscripts describing the process and results of the implementation of an evidencebased nursing intervention. Manuscripts should be no longer than 1500 words, including references. All manuscripts will be peer reviewed and submission does not guarantee publication.

We also welcome letters from our readers about Evidence-Based Nursing. We would like to hear about the positive and negative aspects of our journal. Your feedback is most important in assisting us to produce a high quality journal which is useful to the practising nurse. Letters should be no longer than 400 words.

All submitted material should be typewritten, double spaced, and mailed or faxed to Nicky Cullum in the UK editorial office (Centre for Evidence Based Nursing, University of York, Genesis 6, York Science Park, York YO10 5DQ. Fax: + 44 (0) 1904 43410). 